\title{
KAWAMATA-VIEHWEG VANISHING AS KODAIRA VANISHING FOR STACKS
}

\author{
Kenji Matsuki and Martin Olsson
}

\begin{abstract}
We associate to a pair $(X, D)$, consisting of a smooth variety with a divisor $D \in \operatorname{Div}(X) \otimes \mathbb{Q}$ whose support has only normal crossings, a canonical Deligne-Mumford stack over $X$ on which $D$ becomes integral. We then reinterpret the Kawamata-Viehweg vanishing theorem as Kodaira vanishing for stacks.
\end{abstract}

\section{Introduction}

Let $k$ be a field, and suppose given a pair $(X, D)$, where $X / k$ is a smooth variety and $D \in \operatorname{Div}(X) \otimes \mathbb{Q}$ is a divisor whose support has only normal crossings. Suppose further that there exists an integer $n$, prime to char $(k)$, for which $n D$ is integral. Our aim in this note is to explain how one can associate to $(X, D)$ a Deligne-Mumford stack $\mathcal{X} / X$, which is the "minimal covering" of $X$ on which $D$ becomes integral (see (4.1) for the precise properties of $\mathcal{X}$ ). In addition, we explain, using the stack $\mathcal{X}$, how the Kawamata-Viehweg vanishing theorem ([4], [7], [11]) can naturally be interpreted as an application of Kodaira vanishing for stacks.

There are no new vanishing theorems in this paper, except for explicitly stating a Kodaira vanishing theorem for Deligne-Mumford stacks, following Deligne, Illusie, and Raynaud.

The note is organized as follows. In section 2 we present a Kodaira vanishing theorem for Deligne-Mumford stacks. A Hodge theoretic approach to the characteristic 0 version of this theorem had previously been considered by Starr (private communication). In section 3 we recall some definitions and make a few basic observations concerning log structures in the sense of Fontaine and Illusie which will be needed in the construction of $\mathcal{X}$. In section 4 we construct the stack $\mathcal{X}$ and describe its basic properties. Then in section 5 , we discuss the Kawamata-Viehweg vanishing theorem.

\section{Kodaira vanishing for stacks}

Theorem 2.1. Let $k$ be a field, and let $\mathcal{X} / k$ be a smooth proper tame DeligneMumford stack of dimension d with projective coarse moduli space $\pi: \mathcal{X} \rightarrow X$. Suppose that $\pi$ is flat and let $L$ be an invertible sheaf on $\mathcal{X}$ such that some power of $L$ descends to an ample invertible sheaf on $X$.

Received March 27, 2003. 
(i). If $\operatorname{char}(k)=0$, then $H^{j}\left(\mathcal{X}, \Omega_{\mathcal{X} / k}^{i} \otimes L^{-1}\right)=0$ for $i+j<d$.

(ii). If $\operatorname{char}(k)=p>0, k$ is perfect with ring of Witt vectors $W(k)$, and $\mathcal{X}$ admits a smooth lifting to $W_{2}(k):=W(k) /\left(p^{2}\right)$, then $H^{j}\left(\mathcal{X}, \Omega_{\mathcal{X} / k}^{i} \otimes L^{-1}\right)=0$ for $i+j<\inf (d, p)$.

Theorem (2.1) is proven by the same argument used in the proof by Deligne, Illusie, and Raynaud of the Kodaira vanishing theorem ([2], 2.8). We indicate here only the argument needed for the reduction from characteristic 0 to characteristic $p$, and a basic proposition. The rest of the proof can then be transcribed word for word from (loc. cit.).

Reduction to positive characteristic.

This is fairly standard using the classical argument for schemes ([3]. IV.8.10.5 (xii)). However, we explain the reduction since it uses a non-trivial result about stacks: Chow's lemma for Deligne-Mumford stacks $([9], 16.6 .1)$. So consider $\mathcal{X} / k$ where $k$ is a field of characteristic 0 , and choose a presentation for $\mathcal{X}$. That is, suppose $\mathcal{X}$ is defined by affine schemes $U$ and $R$ together with maps $s, b: R \rightarrow U$ (source and target), $\epsilon: U \rightarrow R$ (identity), $i: R \rightarrow R$ (inverse), $m: R \times_{s, U, b} R \rightarrow R$ (composition), such that the finitely many conditions for these to define an étale groupoid in schemes are satisfied ([9], 2.4.3). Since all these schemes are affine of finite type over $k$, we can find a finitely generated integral $\mathbb{Z}$-algebra $A \subset k$, and an étale groupoid in schemes $(\widetilde{U}, \widetilde{R}, \tilde{s}, \tilde{b}, \tilde{i}, \tilde{m})$ over $A$ inducing our groupoid over $k$. Thus we obtain a Deligne-Mumford stack

$$
g: \tilde{\mathcal{X}} \longrightarrow \operatorname{Spec}(A)
$$

inducing $\mathcal{X}$. Now by $([9], 16.6 .1)$, there exists a projective scheme $Y / k$ together with a proper surjective morphism $f: Y \rightarrow \mathcal{X}$. After localizing on $\operatorname{Spec}(A)$ (and using $([9], 4.18)$ ), we can therefore find a projective scheme $\widetilde{Y}$ and a map $\tilde{f}: \tilde{Y} \rightarrow \tilde{\mathcal{X}}$. Now the condition that the map $\tilde{f}$ is proper and surjective is equivalent to the condition that the induced map of schemes $\widetilde{Y} \times_{\mathcal{X}} \widetilde{U} \rightarrow \widetilde{U}$ is proper and surjective, and hence after localizing on $\operatorname{Spec}(A)$ we can assume that $\tilde{f}$ is proper and surjective. From this it follows that $\tilde{\mathcal{X}}$ is proper over $\operatorname{Spec}(A)$. Let $\tilde{\pi}: \widetilde{\mathcal{X}} \rightarrow \widetilde{X}$ be its coarse moduli space. There is a maximal open substack of $\widetilde{\mathcal{X}}$ which is smooth over $\operatorname{Spec}(A)$ and flat over $\widetilde{X}$, and since $\widetilde{\mathcal{X}}$ is proper over $\operatorname{Spec}(A)$ this implies that after localizing on $\operatorname{Spec}(A)$, we can assume that $\widetilde{\mathcal{X}} / A$ is tame and smooth and that $\tilde{\pi}$ is flat. Now giving the invertible sheaf $L$ on $\mathcal{X}$ is equivalent to giving a morphism $\mathcal{X} \rightarrow B \mathbb{G}_{m}$. Hence by $([9], 4.18)$, we can after further localization on $\operatorname{Spec}(A)$ find a sheaf $\widetilde{L}$ on $\widetilde{\mathcal{X}}$ inducing $L$ such that some tensor power of $\widetilde{L}$ descends to an ample invertible sheaf on $\widetilde{X}$.

Lemma 2.2. Let $\mathcal{X} / S$ be a tame Deligne-Mumford stack of finite type over a noetherian scheme $S$, and suppose the map $\pi: \mathcal{X} \rightarrow X$ from $\mathcal{X}$ to its coarse moduli space is flat. Then

(i). If $F$ is a coherent sheaf on $\mathcal{X}$, then $R^{i} \pi_{*} F=0$ for $i>0$, and the formation of $\pi_{*} F$ is compatible with arbitrary base change $S^{\prime} \rightarrow S$. 
(ii). If $E$ is a locally free sheaf on $\mathcal{X}$, then $\pi_{*} E$ is locally free on $X$.

(iii). If $\mathcal{X}$ is Cohen-Macaulay, then $X$ is Cohen-Macaulay.

Proof. It is well known that étale locally on $X$, the stack $\mathcal{X}$ is isomorphic to $[U / \Gamma]$, where $U=\operatorname{Spec}(R)$ is an affine scheme and $\Gamma$ is a finite group of order invertible in $R$ acting on $U$ (see for example ([1], 2.2.3)). Moreover, in this situation $X=\operatorname{Spec}\left(R^{\Gamma}\right)$. Now if $M$ is any $R^{\Gamma}$-module with action of $\Gamma$, then the invariant $M^{\Gamma}$ is a direct summand of $M$. Indeed, the map

$$
m \longmapsto \frac{1}{|\Gamma|} \sum_{\gamma \in \Gamma} m^{\gamma}
$$

defines a retraction $M \rightarrow M^{\Gamma}$. From this (i) and (ii) follow. Statement (iii) follows from this discussion combined with ([5], remark 2.3).

It follows from the lemma and standard base change theorems for cohomology on projective schemes, that after localizing on $\operatorname{Spec}(A)$, we may assume that the groups $R^{j} g_{*}\left(\Omega_{\tilde{X} / A}^{i} \otimes \widetilde{L}^{-1}\right)$ are locally free on $\operatorname{Spec}(A)$ of constant rank, and compatible with base change. Let $T$ be the scheme-theoretic closure of a closed point of $\operatorname{Spec}(A \otimes \mathbb{Q})$. The scheme $T$ is quasi-finite and flat over $\operatorname{Spec}(\mathbb{Z})$. Choose a closed point $t \in T$ at which $T / \mathbb{Z}$ is étale and for which $\operatorname{char}(k(t))>d$. Then $\widetilde{\mathcal{X}} \otimes k(t)$ admits a lifting to $W_{2}(k(t))$, and so it suffices to prove the theorem for the pair $(\widetilde{\mathcal{X}} \otimes k(t), \widetilde{L} \otimes k(t))$. This concludes the reduction to the positive characteristic case.

Proposition 2.3. Let $k$ be a field, $\mathcal{X} / k$ a smooth tame Deligne-Mumford stack, and $\pi: \mathcal{X} \rightarrow X$ its coarse moduli space. Assume that $X$ is projective and that $\pi$ is flat. Then if $L$ is an invertible sheaf on $\mathcal{X}$ such that some tensor power descends to an ample sheaf on $X$, there exists an integer $N_{0}$ such that $H^{j}\left(\mathcal{X}, \Omega_{\mathcal{X} / k}^{i} \otimes L^{-N}\right)=0$ for $j<d$, all $i$, and all $N \geq N_{0}$.

Proof. Let $b$ be an integer such that $L^{\otimes b}=\pi^{*} M$ for some ample line bundle $M$ on $X$.

Because $X$ is Cohen-Macaulay (2.2 (iii)), $X$ has a dualizing sheaf $\omega_{X}$. Since $M$ is ample, there exists an integer $l_{0}$ such that for $j<d$, all $i, 0 \leq r<b$, and $l \geq l_{0}$

$$
H^{d-j}\left(X, \omega_{X} \otimes\left(\pi_{*}\left(\Omega_{\mathcal{X} / k}^{i} \otimes L^{-r}\right)\right)^{*} \otimes M^{l}\right)=0,
$$

where $\left(\pi_{*}\left(\Omega_{\mathcal{X} / k}^{i} \otimes L^{-r}\right)\right)^{*}$ denotes the dual of the locally free (by $(2.2$ (ii) $\left.)\right)$ sheaf $\pi_{*}\left(\Omega_{\mathcal{X} / k}^{i} \otimes L^{-r}\right)$. We claim that $N_{0}=b l_{0}$ works in the proposition. Indeed, for any $N \geq N_{0}$, write $N=r+b l$, where $0 \leq r<b$ and $l \geq l_{0}$. Then applying $(2.2$ (i)) and Serre duality, we have

$$
H^{j}\left(\mathcal{X}, \Omega_{\mathcal{X} / k}^{i} \otimes L^{-N}\right)=H^{d-j}\left(X, \omega_{X} \otimes\left(\pi_{*}\left(\Omega_{\mathcal{X} / k}^{i} \otimes L^{-r}\right)\right)^{*} \otimes M^{l}\right)^{*}=0
$$

for $j<d$. 


\section{Locally free log structures}

The stack $\mathcal{X}$ in (4.1) will be constructed as a moduli space classifying certain $\log$ structures (in the sense of Fontaine and Illusie $([6])$ ). Thus before giving the construction and proving (4.1) in the next section, we review some basic definitions and results concerning log structures and introduce some terminology. For more details about log structures see ([6]).

Fix a scheme $X$. In what follows all monoids are commutative with unit.

Definition 3.1 ([6], 1.1 and 1.2). A prelog structure on $X$ is a sheaf of monoids $\mathcal{M}$ on the étale site of $X$ together with a map of sheaves of monoids $\alpha: \mathcal{M} \rightarrow \mathcal{O}_{X}$, where $\mathcal{O}_{X}$ is viewed as a monoid under multiplication. We usually abuse notation and write simply $\mathcal{M}$ for the prelog structure $(\mathcal{M}, \alpha)$. A prelog structure $\mathcal{M}$ is a $\log$ structure if the map $\alpha^{-1}\left(\mathcal{O}_{X}^{*}\right) \rightarrow \mathcal{O}_{X}^{*}$ is an isomorphism. A morphism of (pre-) $\log$ structures $(\mathcal{N}, \beta) \rightarrow(\mathcal{M}, \alpha)$ is a map $\rho: \mathcal{N} \rightarrow \mathcal{M}$ of sheaves of monoids such that $\alpha \circ \rho=\beta$.

Observe that for a $\log$ structure $\alpha: \mathcal{M} \rightarrow \mathcal{O}_{X}$, the isomorphism $\alpha^{-1}\left(\mathcal{O}_{X}^{*}\right) \simeq$ $\mathcal{O}_{X}^{*}$ identifies the sheaf of invertible elements in $\mathcal{M}$ with $\mathcal{O}_{X}^{*}$. We denote by $\overline{\mathcal{M}}$ the quotient of $\mathcal{M}$ by $\mathcal{O}_{X}^{*}$.

By $([6], 1.3)$, the inclusion functor

$$
(\log \text { structures on } X) \rightarrow(\text { prelog structures on } X)
$$

has a left adjoint which we denote by $\mathcal{N} \mapsto \mathcal{N}^{a}$. The log structure $\mathcal{N}^{a}$ is called the log structure associated to $\mathcal{N}$. Explicitly for a prelog structure $\alpha: \mathcal{N} \rightarrow \mathcal{O}_{X}$ the $\log$ structure $\mathcal{N}^{a}$ is obtained as the pushout of the diagram

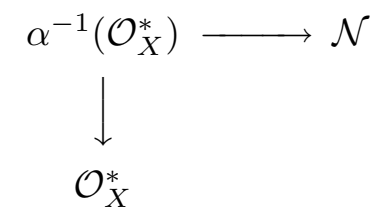

with the natural map $\mathcal{N}^{a} \rightarrow \mathcal{O}_{X}$. A log structure $\mathcal{M}$ on $X$ is called fine if étale locally on $X$ there exists a finitely generated integral monoid $P$ and a map of monoids $P \rightarrow \Gamma(X, \mathcal{M})$ such that the induced map $\underline{P} \rightarrow \mathcal{M}$ from the constant sheaf $\underline{P}$ identifies $\mathcal{M}$ with the $\log$ structure associated to the prelog structure

$$
\underline{P} \rightarrow \mathcal{M} \rightarrow \mathcal{O}_{X}
$$

Such a map $P \rightarrow \Gamma(X, \mathcal{M})$ is called a chart for $\mathcal{M}$.

Example 3.2 ([6], 1.5 and 2.5). Let $X / k$ be a smooth scheme over a field $k$ and let $D \subset X$ be a divisor with normal crossings. Define $\mathcal{M} \subset \mathcal{O}_{X}$ to be the subsheaf of monoids which to any étale map $U \rightarrow X$ associates the monoid of functions $f \in \mathcal{O}_{X}(U)$ whose restriction to $U \times_{X}(X-D)$ is invertible. Then $\mathcal{M}$ is a $\log$ structure on $X$. Moreover, by our assumptions there exists étale locally on $X$ an étale morphism

$$
X \rightarrow \operatorname{Spec}\left(k\left[x_{1}, \ldots, x_{n}\right]\right)
$$


for some $n$, such that $D$ is equal to the inverse image of the divisor $\left\{x_{1} \cdots x_{r}=0\right\}$ for some $r \leq n$. In this local situation, the $\log$ structure $\mathcal{M}$ can be described as the $\log$ structure associated to the chart $\mathbb{N}^{r} \rightarrow \mathcal{O}_{X}$ sending the $i$-th generator $e_{i}$ of $\mathbb{N}^{r}$ to $x_{i}$. In particular, $\mathcal{M}$ is a fine $\log$ structure on $X$.

The following result is a key technical tool used to construct charts:

Lemma 3.3 ([6], 2.10). Let $\mathcal{M}$ be a fine log structure on $X$ and $\bar{x} \rightarrow X$ a geometric point. Assume given a surjection of groups $G \rightarrow \overline{\mathcal{M}}_{\bar{x}}^{\mathrm{gp}}$ (where $\overline{\mathcal{M}}_{\bar{x}} \rightarrow$ $\overline{\mathcal{M}}_{\bar{x}}^{\mathrm{gp}}$ denotes the universal map from $\overline{\mathcal{M}}_{\bar{x}}$ to a group) and set $P:=G \times \overline{\mathcal{M}}_{\bar{x}}^{\mathrm{gp}} \overline{\mathcal{M}}_{\bar{x}}$. Then any lifting $P \rightarrow \mathcal{M}_{\bar{x}}$ of the projection $P \rightarrow \overline{\mathcal{M}}_{\bar{x}}$ extends to a chart for $\mathcal{M}$ in some étale neighborhood of $\bar{x}$.

Remark 3.4. In the above Lemma, and in the discussion below, we freely use the notion of the stalk of an étale sheaf $\mathcal{F}$ at a geometric point $\bar{x} \rightarrow X$. Recall that for such a sheaf $\mathcal{F}$, the stalk $\mathcal{F}_{\bar{x}}$ is the $\operatorname{limit} \underline{\lim } \mathcal{F}(U)$ taken over the inductive system of commutative diagrams

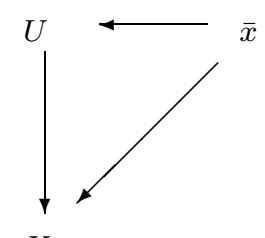

where $U / X$ is étale. In particular, the stalk $\mathcal{O}_{X, \bar{x}}$ denotes the strict henselization of the local ring of $X$ at the image of $\bar{x}$.

Recall that if $P$ is a unit free monoid (i.e. the unit element $0 \in P$ is the only invertible element in $P$ ), then an element $p \in P$ is called irreducible if for every $p_{1}, p_{2} \in P$ for which $p=p_{1}+p_{2}$, either $p_{1}=p$ or $p_{2}=p$. We denote the set of irreducible elements in $P$ by $\operatorname{Irr}(P)$.

Definition 3.5. (i). A monoid $F$ is called free if it is isomorphic to $\mathbb{N}^{r}$ for some $r$. The integer $r$ is uniquely determined and is called the rank of $F$.

(ii). A morphism $\varphi: F_{1} \rightarrow F_{2}$ between free monoids is called simple if $F_{1}$ and $F_{2}$ have the same rank, $\varphi$ is injective, and if for every irreducible element $f_{1} \in F_{1}$ there exists an irreducible element $f_{2} \in F_{2}$ and an integer $n$ such that $n f_{2}=\varphi\left(f_{1}\right)$.

(iii). A locally free log structure on $X$ is a fine $\log$ structure $\mathcal{M}$ on $X$ such that for every geometric $\bar{x} \rightarrow X$ the monoid $\overline{\mathcal{M}}_{\bar{x}}$ is free.

(iv). A morphism $\varphi: \mathcal{M}_{1} \rightarrow \mathcal{M}_{2}$ between locally free log structures on $X$ is called simple if for every geometric point $\bar{x} \rightarrow X$, the map $\varphi_{\bar{x}}: \overline{\mathcal{M}}_{1, \bar{x}} \rightarrow \overline{\mathcal{M}}_{2, \bar{x}}$ is simple in the sense of (ii) above.

Remark 3.6. If $\varphi: F_{1} \rightarrow F_{2}$ is a simple morphism as in (3.5 (ii)), then for each $f_{1} \in \operatorname{Irr}\left(F_{1}\right)$, the element $f_{2} \in \operatorname{Irr}\left(F_{2}\right)$ for which there exists an integer $n$ such that $n f_{2}=\varphi\left(f_{1}\right)$ is uniquely determined. From this it follows that $\varphi$ induces a canonical bijection $\operatorname{Irr}\left(F_{1}\right) \rightarrow \operatorname{Irr}\left(F_{2}\right)$. 
Lemma 3.7. Let $\varphi: \mathcal{M}_{1} \rightarrow \mathcal{M}_{2}$ be a simple morphism of locally free log structures on $X$, and let $\beta_{1}: \mathbb{N}^{r} \rightarrow \mathcal{M}_{1}$ be a chart for $\mathcal{M}_{1}$. Let $\bar{x} \rightarrow X$ be a geometric point, and suppose given a surjective map $\bar{\beta}_{2}: \mathbb{N}^{r} \rightarrow \overline{\mathcal{M}}_{2, \bar{x}}$ and integers $\left\{b_{i}\right\}_{i=1}^{r}$ prime to the characteristic of $k(\bar{x})$ such that the diagram

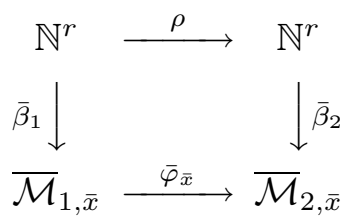

commutes, where $\rho:=\oplus\left(\times b_{i}\right)$. Then in some étale neighborhood of $\bar{x}$, there exists a chart $\beta_{2}: \mathbb{N}^{r} \rightarrow \mathcal{M}_{2}$ lifting $\bar{\beta}_{2}$ such that the diagram

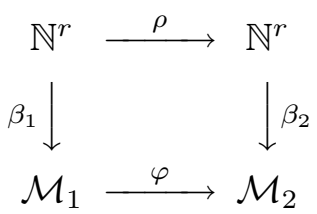

commutes.

Proof. By (3.3), it suffices to find a lifting $\beta_{2}: \mathbb{N}^{r} \rightarrow \mathcal{M}_{2, \bar{x}}$ lifting $\bar{\beta}_{2}$ such that the diagram

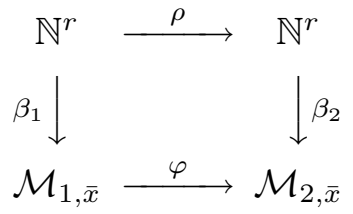

commutes. For this, choose for each $1 \leq i \leq r$ a lifting $m_{i} \in \mathcal{M}_{2, \bar{x}}$ of $\bar{\beta}_{2}\left(e_{i}\right)$, where $e_{i}$ denotes the $i$-th standard generator for $\mathbb{N}^{r}$. By the commutativity of (3.7.1), there exists a unit $u_{i} \in \mathcal{O}_{X, \bar{x}}^{*}$ such that $\lambda\left(u_{i}\right)+b_{i} m_{i}=\varphi\left(\beta_{1}\left(e_{i}\right)\right)$, where $\lambda: \mathcal{O}_{X, \bar{x}}^{*} \rightarrow \mathcal{M}_{X, \bar{x}}$ denotes the canonical inclusion. Since by assumption $b_{i}$ is invertible on $X$, there exists a unit $v_{i} \in \mathcal{O}_{X, \bar{x}}^{*}$ such that $v_{i}^{b_{i}}=u_{i}$. Replacing $m_{i}$ by $m_{i}+\lambda\left(v_{i}\right)$, we can therefore find units $m_{i} \in \mathcal{M}_{2, \bar{x}}$ such that $b_{i} m_{i}=\varphi\left(\beta_{1}\left(e_{i}\right)\right)$. That is, a map $\beta_{2}: \mathbb{N}^{r} \rightarrow \mathcal{M}_{2, \bar{x}}$ such that (3.7.3) commutes.

\section{Construction of $\mathcal{X}$}

Theorem 4.1. Let $X / k$ be a smooth scheme over a field $k, D=\bigcup_{i \in I} D_{i} \subset X$ a divisor with normal crossings, and $\left\{b_{i}\right\}_{i \in I}$ a collection of positive integers prime to $\operatorname{char}(k)$. Attached to this data is a canonical pair $\left(\mathcal{X}, \widetilde{D}=\bigcup_{i \in I} \widetilde{D}_{i}\right)$, consisting of a smooth tame Deligne-Mumford stack $\pi: \mathcal{X} \rightarrow X$ together with a normal crossings divisor $\widetilde{D} \subset \mathcal{X}$ such that:

(i). The map $\pi$ is finite and flat, identifies $X$ with the coarse moduli space of $\mathcal{X}$, and is an isomorphism over $X-D$.

(ii). The pullback $\pi^{*} \mathcal{O}_{X}\left(-D_{i}\right)$ is equal to $\mathcal{O}_{\mathcal{X}}\left(-b_{i} \widetilde{D}_{i}\right)$ as a subsheaf of $\mathcal{O}_{\mathcal{X}}$. 
(iii). For any collection $\left\{a_{i}\right\}_{i \in I}$ of positive integers with $b_{i} \nmid a_{i}$, there is a natural quasi-isomorphism

$$
\Omega_{X / k}^{j}(\log D) \otimes \mathcal{O}_{X}\left(-\sum_{i}\left\ulcorner a_{i} / b_{i}\right\urcorner D_{i}\right) \longrightarrow R \pi_{*}\left(\Omega_{\mathcal{X} / k}^{j} \otimes \mathcal{O}_{\mathcal{X}}\left(-\sum_{i} a_{i} \widetilde{D}_{i}\right)\right),
$$

where for $q \in \mathbb{Q}$, we denote by $\ulcorner q\urcorner:=\inf \{p \in \mathbb{Z} \mid p \geq q\}$.

(iv). Let $\mathcal{M}_{X}$ denote the log structure on $X$ associated to the divisor $D$, and suppose $A \rightarrow k$ is a surjective map from an Artin local ring $A$. Then associated to any log smooth lifting $\left(\widetilde{X}, \mathcal{M}_{X}\right)$ of $\left(X, \mathcal{M}_{X}\right)$ to $\operatorname{Spec}(A)$, there is a canonical smooth lifting $\tilde{\mathcal{X}}$ of $\mathcal{X}$ to $\operatorname{Spec}(A)$.

(v). If $f: Y \rightarrow X$ is a finite flat morphism from a smooth variety $Y / k$ for which $f^{*}\left(\sum_{i} \frac{1}{b_{i}} D_{i}\right)$ is integral with support a divisor with normal crossings, then there exists a unique map $Y \rightarrow \mathcal{X}$ over $X$.

Proof. Let $\mathcal{M}_{X}$ be the locally free $\log$ structure associated to the divisor $D$ as in (3.2). If $\bar{x} \rightarrow X$ is a geometric point, let $C(\bar{x})$ denote the irreducible components of the inverse image of $D$ in $\operatorname{Spec}\left(\mathcal{O}_{X, \bar{x}}\right)$.

Lemma 4.2. For every geometric point $\bar{x} \rightarrow X$ there is a canonical isomorphism $\overline{\mathcal{M}}_{X, \bar{x}} \simeq \bigoplus_{C(\bar{x})} \mathbb{N}$.

Proof. It suffices to establish a bijection $\operatorname{Irr}\left(\overline{\mathcal{M}}_{X, \bar{x}}\right) \rightarrow C(\bar{x})$. We do so by sending an irreducible element $\bar{m}$ to the component defined by the ideal $(\alpha(m))$, where $m \in \mathcal{M}_{X, \bar{x}}$ is any lifting of $m$ and $\alpha: \mathcal{M}_{X, \bar{x}} \rightarrow \mathcal{O}_{X, \bar{x}}$ is the logarithm map. That this is well-defined and a bijection can be verified after replacing $X$ by an étale cover. Hence it suffices to consider the case when $x$ is the point $x_{1}=\cdots=x_{n}=0$ on $X=\operatorname{Spec}\left(k\left[x_{1}, \ldots, x_{n}\right]\right)$ and $D=Z\left(x_{1} \cdots x_{r}\right)$, for some $n$ and $r$. In this case the result is clear.

If $f: Y \rightarrow X$ is any morphism of schemes, and $f^{*} \mathcal{M}_{X} \rightarrow \mathcal{M}$ is a simple morphism on $Y$, where $\mathcal{M}_{X}$ and $\mathcal{M}$ are locally free log structures on $X$ and $Y$ respectively, then for every geometric point $\bar{y} \rightarrow Y$ with image $\bar{x}=f(\bar{y})$, the map

$$
\overline{\mathcal{M}}_{X, \bar{x}} \longrightarrow \overline{\mathcal{M}}_{\bar{y}}
$$

has by (4.2) and (3.6) a canonical decomposition

$$
\oplus\left(\times c_{i}\right): \bigoplus_{C(\bar{x})} \mathbb{N} \longrightarrow \bigoplus_{C(\bar{x})} \mathbb{N}
$$

where $\left\{c_{i}\right\}$ is a collection of positive integers indexed by $C(\bar{x})$.

Define $\pi: \mathcal{X} \rightarrow X$ to be the fibered category whose fiber $\mathcal{X}(Y)$ over $f: Y \rightarrow X$ is the groupoid of simple morphisms of $\log$ structures $\varphi: f^{*} \mathcal{M}_{X} \rightarrow \mathcal{M}$, such that for each geometric point $\bar{y} \rightarrow Y$ with $\bar{x}=f(\bar{y})$, the integer $c_{i}$ associated to a component $C_{i} \in C(\bar{x})$ is equal to the integer $b_{i}$ attached (by our assumptions) 
to the component $D_{i} \subset X$ containing the image of $C_{i}$ under the natural map $\operatorname{Spec}\left(\mathcal{O}_{X, \bar{x}}\right) \rightarrow X$. A morphism

$$
\rho:\left(\varphi_{1}: f^{*} \mathcal{M}_{X} \rightarrow \mathcal{M}_{1}\right) \longrightarrow\left(\varphi_{2}: f^{*} \mathcal{M}_{X} \rightarrow \mathcal{M}_{2}\right)
$$

in $\mathcal{X}(Y)$ is an isomorphism of $\log$ structures $\rho: \mathcal{M}_{1} \rightarrow \mathcal{M}_{2}$ such that $\rho \circ \varphi_{1}=\varphi_{2}$. With the natural notion of pullback, $\mathcal{X}$ is a fibered category. Moreover, because $\mathcal{X}$ classifies étale sheaves, $\mathcal{X}$ is a stack with respect to the étale topology.

Lemma 4.3. The stack $\mathcal{X}$ is a Deligne-Mumford stack. If $X$ is equal to $\operatorname{Spec}\left(k\left[x_{1}, \ldots, x_{n}\right]\right)$ and $D_{i}=Z\left(x_{i}\right)$ for $1 \leq i \leq r$, then $\mathcal{X}$ is canonically isomorphic to

$$
\left[\operatorname{Spec}\left(k\left[y_{1}, \ldots, y_{n}\right]\right) / \mu_{b_{1}} \times \cdots \times \mu_{b_{r}}\right],
$$

where $k\left[y_{1}, \ldots, y_{n}\right]$ is viewed as a $k\left[x_{1}, \ldots, x_{n}\right]$-algebra via the map induced by

$$
x_{i} \mapsto\left\{\begin{array}{cl}
y_{i}^{b_{i}} & \text { if } i \leq r \\
y_{i} & \text { if } i>r
\end{array}\right.
$$

and $\mu_{b_{1}} \times \cdots \times \mu_{b_{r}}$ acts by

$$
\left(u_{1}, \ldots, u_{r}\right) \cdot y_{i}=\left\{\begin{array}{cl}
u_{i} y_{i} & \text { if } i \leq r \\
y_{i} & \text { if } i>r .
\end{array}\right.
$$

Proof. Since $\mathcal{X}$ is a stack with respect to the étale topology, the assertion that $\mathcal{X}$ is a Deligne-Mumford stack can be verified étale locally on $X$. Thus it suffices to prove the second statement. Let us temporarily denote the stack (4.3.1) by $\mathcal{X}^{\prime}$. By $([10], 5.20), \mathcal{X}^{\prime}$ is canonically isomorphic to the stack which to any $f: Y \rightarrow X$ associates the groupoid of morphisms of log structures $f^{*} \mathcal{M}_{X} \rightarrow \mathcal{M}$ together with a map $\bar{\beta}^{\prime}: \mathbb{N}^{r} \rightarrow \overline{\mathcal{M}}$ which étale locally lifts to a chart, such that the diagram

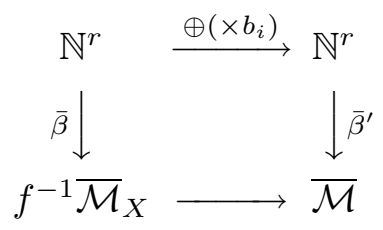

commutes, where $\bar{\beta}$ denotes the map induced by the standard chart on $\mathcal{M}_{X}$. It follows that there is a natural functor $F: \mathcal{X}^{\prime} \rightarrow \mathcal{X}$ which simply forgets the additional data of the map $\bar{\beta}^{\prime}$. By (3.7), every object of $\mathcal{X}(Y)$ is étale locally in the essential image of $F$, so to prove that $F$ is an equivalence, it suffices to prove that it is fully faithful. This amounts to the following statement. Given two objects $\left(\varphi_{i}: f^{*} \mathcal{M}_{X} \rightarrow \mathcal{M}_{i}, \beta_{i}^{\prime}\right)$ of $\mathcal{X}^{\prime}(Y)$, any isomorphism $\rho: \mathcal{M}_{1} \rightarrow \mathcal{M}_{2}$, such that $\rho \circ \varphi_{1}=\varphi_{2}$, has the property that $\bar{\rho} \circ \bar{\beta}_{1}^{\prime}=\bar{\beta}_{2}^{\prime}$, where $\bar{\rho}: \overline{\mathcal{M}}_{1} \rightarrow \overline{\mathcal{M}}_{2}$ denotes the map induced by $\rho$. But this is clear, for since $\overline{\mathcal{M}}_{2}$ has no units, two maps $\mathbb{N}^{r} \rightarrow \overline{\mathcal{M}}_{2}$ are equal if and only if their restrictions to the image of $\oplus\left(\times b_{i}\right)$ are equal. Thus $F$ is fully faithful, and the lemma follows. 
We can now complete the proof of (4.1). We define $\widetilde{D}_{i}$ to be the unique reduced closed substack of $\mathcal{X}$ whose support is equal to the support of the closed substack defined by $\pi^{*} \mathcal{O}_{X}\left(-D_{i}\right)$. By the local description of $\mathcal{X}$ given above, $\widetilde{D}:=\bigcup \widetilde{D}_{i}$ is a reduced divisor with normal crossings, and (4.1 (ii)) holds. Also the local description of $\mathcal{X}$ in (4.3) implies that $X$ is the coarse moduli space of $\mathcal{X}$ (see for example ([1], 2.3.3)).

To see $(4.1(\mathrm{iii}))$, note first that since $\Omega_{\mathcal{X} / k}^{j}(\log \widetilde{D})=\pi^{*} \Omega_{X / k}^{j}(\log D)$ and $a_{i}<b_{i}\left\ulcorner a_{i} / b_{i}\right\urcorner$ for all $i$ (since $b_{i} \nmid a_{i}$ ), there is a natural map

$$
\pi^{*}\left(\Omega_{X / k}^{j}(\log D) \otimes \mathcal{O}_{X}\left(-\sum_{i}\left\ulcorner a_{i} / b_{i}\right\urcorner D_{i}\right)\right) \longrightarrow \Omega_{\mathcal{X} / k}^{j} \otimes \mathcal{O}_{\mathcal{X}}\left(-\sum_{i} a_{i} \widetilde{D}_{i}\right),
$$

which by adjoint induces a map

$$
\Omega_{X / k}^{j}(\log D) \otimes \mathcal{O}_{X}\left(-\sum_{i}\left\ulcorner a_{i} / b_{i}\right\urcorner D_{i}\right) \longrightarrow \pi_{*}\left(\Omega_{\mathcal{X} / k}^{j} \otimes \mathcal{O}_{\mathcal{X}}\left(-\sum_{i} a_{i} \widetilde{D}_{i}\right)\right) .
$$

Moreover, $R^{i} \pi_{*}\left(\Omega_{\mathcal{X} / k}^{j} \otimes \mathcal{O}_{\mathcal{X}}\left(-\sum_{i} a_{i} \widetilde{D}_{i}\right)\right)=0$ for $i>0$ by $(2.2(\mathrm{i}))$, so there is a natural quasi-isomorphism

$$
\pi_{*}\left(\Omega_{\mathcal{X} / k}^{j} \otimes \mathcal{O}_{\mathcal{X}}\left(-\sum_{i} a_{i} \widetilde{D}_{i}\right)\right) \simeq R \pi_{*}\left(\Omega_{\mathcal{X} / k}^{j} \otimes \mathcal{O}_{\mathcal{X}}\left(-\sum_{i} a_{i} \widetilde{D}_{i}\right)\right) .
$$

Thus to show (4.1 (iii)), it suffices to show that the map (4.4.2) is an isomorphism. This can be verified étale locally on $X$, and so we need only consider $X$ and $\mathcal{X}$ as in (4.3). In this case, the assertion amounts to the statement that a $j$-form

$$
y_{1}^{\alpha_{1}} \cdots y_{r}^{\alpha_{r}} d y_{i_{1}} \wedge \cdots \wedge d y_{i_{j}}
$$

with $\alpha_{i} \geq a_{i}$ for all $i$, is invariant under the action of $\mu_{b_{1}} \times \cdots \times \mu_{b_{r}}$ if and only if $b_{i} \mid \alpha_{i}$ and $\alpha_{i} / b_{i} \geq\left\ulcorner a_{i} / b_{i}\right\urcorner$ for $i \notin\left\{i_{1}, \ldots, i_{j}\right\}$ and $b_{i} \mid\left(\alpha_{i}+1\right)$ and $\left(\alpha_{i}+1\right) / b_{i} \geq$ $\left\ulcorner a_{i} / b_{i}\right\urcorner$ for $i \in\left\{i_{1}, \ldots, i_{j}\right\}$. This follows immediately from the definition of the action.

As for $(4.1$ (iv)), let $\widetilde{\mathcal{X}}$ be the fibered category on the category of $\widetilde{X}$-schemes, which to any $f: Y \rightarrow \widetilde{X}$ associates the groupoid of morphisms of log structures $f^{*} \mathcal{M}_{X} \rightarrow \mathcal{M}$ which when pulled back to $Y \otimes_{A} k$ defines an object of $\mathcal{X}\left(Y \otimes_{A} k\right)$. Since $\left(\tilde{X}, \mathcal{M}_{X}\right)$ is $\log$ smooth over $\operatorname{Spec}(A)$, it follows from the structure theorem for $\log$ smooth morphisms $([6], 3.4)$ that étale locally on $\widetilde{X}$, the log scheme $\left(\widetilde{X}, \mathcal{M}_{\tilde{X}}\right)$ is isomorphic to $\operatorname{Spec}\left(A\left[x_{1}, \ldots, x_{n}\right]\right)$ with log structure defined by

$$
\mathbb{N}^{r} \longrightarrow A\left[x_{1}, \ldots, x_{n}\right], \quad\left(i_{1}, \ldots, i_{r}\right) \mapsto x_{1}^{i_{1}} \cdots x_{r}^{i_{r}},
$$

for some $n$ and $r$. From this and the same argument used in the proof of (4.3), it follows that $\widetilde{\mathcal{X}}$ is a smooth lifting of $\mathcal{X}$.

To prove $(4.1(\mathrm{v}))$, we have to attach to any $f: Y \rightarrow X$ as in the theorem, a unique (up to unique isomorphism) object $f^{*} \mathcal{M}_{X} \rightarrow \mathcal{M}$ of $\mathcal{X}(Y)$. Since $f$ is flat, $f^{*} \mathcal{M}_{X}$ is a subsheaf of $\mathcal{O}_{Y}$. From this it follows that $\mathcal{M}$ is also a subsheaf of $\mathcal{O}_{Y}$. Moreover, we can completely characterize the sheaf $\mathcal{M}$ as follows. Etale 
locally on $X$, we can find a chart $\mathbb{N}^{r} \rightarrow \mathcal{M}_{X}$ for the log structure on $X$. For each generator $e_{i} \in \mathbb{N}^{r}$, let $f_{i}$ be its image in $\mathcal{O}_{X}$ and let $b_{i}$ denote the corresponding integer attached to the component of $D$ defined by $f_{i}$. By assumption, there exists étale locally on $Y$ an element $g_{i} \in \mathcal{O}_{Y}$, well defined up to multiplication by a root of unity, with $g_{i}^{b_{i}}=f_{i}$. The $\log$ structure $\mathcal{M}$ is then the subsheaf of monoids of $\mathcal{O}_{Y}$ generated by the $g_{i}$ and $\mathcal{O}_{Y}^{*}$. This also proves the existence.

\section{The Kawamata-Viehweg vanishing theorem}

Let $k$ be a field, $X / k$ be a smooth projective variety of pure dimension $d$, and $E=\sum_{i} a_{i} D_{i} \in \operatorname{Div}(X) \otimes \mathbb{Q}$ a $\mathbb{Q}$-divisor, where the $D_{i}$ are the distinct irreducible components. Define the integral part $[E]$, the round up $\ulcorner E\urcorner$, and the fractional part $\langle E\rangle$ by

$$
\begin{aligned}
{[E] } & :=\sum\left[a_{i}\right] D_{i} \\
\ulcorner E\urcorner & :=\sum\left\ulcorner a_{i}\right\urcorner D_{i}=-[-E] \\
\langle E\rangle & :=\sum\left\langle a_{i}\right\rangle D_{i}=E-[E],
\end{aligned}
$$

where for $r \in \mathbb{R}$ we write $[r]:=\max \{t \in \mathbb{Z} \mid t \leq r\}$. We suppose that the support of $\langle E\rangle$ is a divisor $D$ with normal crossings, and denote by $\mathcal{M}_{X}$ the associated log structure on $X$ (3.2). The following version of the Kawamata-Viehweg vanishing theorem can be found in $([8], 1-2-2)$ :

Theorem 5.1. Let $X / k$ and $E \in \operatorname{Div}(X) \otimes \mathbb{Q}$ be as above, and assume in addition that $E$ is ample.

(i). Suppose $\operatorname{char}(k)=0$. Denote by $\Omega_{X}^{1}(\log D)$ the sheaf of differentials on $X$ with $\log$ poles along $D$, and by $\Omega_{X}^{i}(\log D)$ its $i$-th exterior power. Then

$$
H^{j}\left(X, \Omega_{X}^{i}(\log D) \otimes \mathcal{O}_{X}(-\ulcorner E\urcorner)\right)=0 \text { for } i+j<d .
$$

(ii). Suppose $k$ is perfect and let $W_{2}(k)$ denote the reduction modulo $p^{2}$ of the ring of Witt vectors of $k$. Suppose further that the smooth log scheme $\left(X, \mathcal{M}_{X}\right)$ admits a log smooth lifting to $W_{2}(k)$. Then

$$
H^{j}\left(X, \Omega_{X}^{i}(\log D) \otimes \mathcal{O}_{X}(-\ulcorner E\urcorner)\right)=0 \text { for } i+j<\inf (d, p) .
$$

Proof. Write $\langle E\rangle=\sum_{i}\left(a_{i} / b_{i}\right) D_{i}$ with $\left(a_{i}, b_{i}\right)=1$. Note that by the openness of the ample cone, we can replace the rational numbers $a_{i} / b_{i}$ by any other rational numbers sufficiently close to the $a_{i} / b_{i}$ without changing the statement of the theorem. Thus in the positive characteristic case, we may assume that the integers $b_{i}$ are prime to $p$. Let $\pi: \mathcal{X} \rightarrow X$ be the stack associated to the data $\left(X, D,\left\{b_{i}\right\}\right)$ as in (4.1). Then by $(4.1$ (iii) $)$, and the projection formula, we have

$$
H^{j}\left(X, \Omega_{X}^{i}(\log D) \otimes \mathcal{O}_{X}(-\ulcorner E\urcorner)\right)=H^{j}\left(\mathcal{X}, \Omega_{\mathcal{X} / k}^{i} \otimes \mathcal{O}_{\mathcal{X}}\left(-\pi^{*}[E]-\sum_{i} a_{i} \widetilde{D}_{i}\right)\right) .
$$

Since $\left(\prod_{i} b_{i}\right) \mathcal{O}_{\mathcal{X}}\left(\pi^{*}[E]+\sum_{i} a_{i} \widetilde{D}_{i}\right)$ descends to an ample sheaf on $X$, the theorem therefore follows from $(2.1)$. 
Remark 5.2. The above theorem plays a crucial and indispensible role in carrying out the proofs for many key ingredients of the so-called Minimal Model Program (MMP for short). The call for Kawamata-Viehweg vanishing, going beyond the classical Kodaira vanishing, is more apparent when we have to deal with the singularities that MMP inevitably brings in higher dimensions ( $\operatorname{dim} \geq 3$ ). This is usually perceived as a technical calamity rather than an indication of any essential point. We wonder and/or speculate, however, that, once we have an interpretation of Kawamata-Viehweg vanishing as a version of Kodaira vanishing for stacks as in this note, there may be some smooth stacks floating around behind the whole game of MMP, and the singularities of MMP we only observe as we look at the coarse moduli of these smooth stacks.

\section{Acknowledgements}

We would like to thank D. Arapura, Y. Kawamata, T. Pantev, and J. Starr for their comments and help. Matsuki was partially supported by NSF grant \#0100598-DMS, and Olsson was partially supported by an NSF post-doctoral research fellowship.

\section{References}

[1] D. Abramovich and A. Vistoli, Compactifying the space of stable maps, J. Amer. Math. Soc 15 (2002), 27-75.

[2] P. Deligne and L. Illusie, Relévements modulo $p^{2}$ et décomposition du complexe de de Rham, Invent. Math. 89 (1987), 247-270.

[3] J. Dieudonné and A. Grothendieck, Éléments de géométrie algébrique, Inst. Hautes Études Sci. Publ. Math. 4, 8, 11, 17, 20, 24, 28, 32 (1961-1967).

[4] H. Esnault and E. Viehweg, Lectures on vanishing theorems, DMV Seminar Band 20 (1992).

[5] M. Hochster and J. Roberts, Rings of invariants of reductive groups acting on regular rings are Cohen-Macaulay, Advances in Math. 13 (1974), 115-175.

[6] K. Kato, Logarithmic structures of Fontaine-Illusie, Algebraic analysis, geometry, and number theory (Baltimore, MD, 1988), Johns Hopkins Univ. Press, Baltimore, MD (1989), 191-224.

[7] Y. Kawamata, A generalization of Kodaira-Ramanujam's vanishing theorem, Math. Ann. 261 (1982), 43-46.

[8] Y. Kawamata, K. Matsuda, and K. Matsuki, Introduction to the minimal model program, Advanced Studies in Pure Math 10 (Algebraic geometry, Sendai 1985), 283-360, NorthHolland, Amsterdam (1987).

[9] G. Laumon and L. Moret-Bailly, Champs algébriques, Ergebnisse der Mathematik 39, Springer-Verlag, Berlin (2000).

[10] M. Olsson, Logarithmic geometry and algebraic stacks, Ann. Sci. École Norm. Sup. 36 (2003), 747-791.

[11] E. Viehweg, Vanishing theorems, J. reine angew. Math. 335 (1982), 1-8.

Department of Mathematics, Purdue University, West Lafayette, IN 47907

E-mail address: kmatsuki@math.purdue.edu

School of Mathematics, Institute for Advanced Study, Princeton, NJ 08540

E-mail address: molsson@ias.edu 